\title{
Maxillary Antrostomy
}

National Cancer Institute

\section{Source}

National Cancer Institute. Maxillary Antrostomy. NCI Thesaurus. Code C51886.

Surgical creation of an opening into the maxillary antrum. 